\title{
Reifegrad von Hochschulen für Future Skills
}

Wie gut schaffen es Hochschulen bereits heute, ihre Studierenden bei der Entwicklung von Future Skills zu unterstützen? In der NextSkills Studie wurden Expertinnen und Experten befragt, wie fit Hochschulen im Bereich der Future Skills sind. Die Delphi-Befragten erhielten dazu eine Liste aller Future Skills inklusive ihrer Beschreibungen und wurden gebeten, deren Wichtigkeit für zukünftige Hochschulbildung einzuschätzen. Ferner sollten sie angeben, inwieweit Hochschulen derzeit in der Lage seien, diese Skills bei ihren Studierenden zu fördern. Beide Variablen wurden jeweils auf einer 5-stufigen Likert-Skala erhoben, die zur Messung der Wichtigkeit von 5 = „sehr wichtig“ bis 1 = „unwichtig“ und für die momentane Fähigkeit der Hochschulen diese Skills auszubilden von $5=$ „sehr gut“ bis $1=$ „sehr gering“ reichte. Um einen Überblick über die möglichen Diskrepanzen zwischen Wichtigkeit eines Skills und Reifegrad dessen aktueller Förderung durch Hochschulen zu erhalten, wurde das Delta der beiden Mittelwerte dieser Variablen berechnet.

\section{A 4.1 Reifegrad für subjekt-entwicklungsbezogene Kompetenzen}

Unter subjekt-entwicklungsbezogenen Kompetenzen werden diejenigen Skills verstanden, die ein Individuum dazu befähigen, auf Sachverhalte zu reagieren, die mit ihm/ ihr selbst zu tun haben - so beispielsweise Reflexion, Autonomie, Selbstwirksamkeit, etc. Alle subjekt-entwicklungsbezogenen Kompetenzen wurden vom Sample der Befragten als wichtig eingestuft, wobei Autonomie (Selbstbestimmungskompetenz) und die Fähigkeit zur Reflexion (Reflexionskompetenz) als sogar sehr wichtig erachtet wurden $\left(M_{\text {Autonomy }}=4,53, S D_{\text {Autonomy }}=0,62 ; M_{\text {Ability to reflect }}=4.50\right.$, $\left.S D_{\text {Ability to reflect }}=0,67\right)$. Außerdem zeigten die Daten, dass die Reflexionskompetenz, zusammen mit Selbstwirksamkeit und Leistungsbereitschaft/-wille (Initiativ- und 
Leistungskompetenz) - im Vergleich zu den anderen subjekt-entwicklungsbezogenen Kompetenzen - die momentan am besten durch die Hochschulen geförderten Future Skills darstellen. Die Future Skill Profile Lernkompetenz $(M=4,48, S D=$ $0,69)$ und Entscheidungskompetenz $(M=4,46, S D=0,72)$ belegten Platz zwei und drei im Ranking der Wichtigkeit. Dies kontrastiert jedoch in hohem Maße mit der Einschätzung zum Reifegrad der Hochschulen, diese Skills bei ihren Studierenden zu fördern: Das hierfür berechnete Delta (siehe Abbildungen 15 und 16) zeigt, dass sich die höchste Diskrepanz zwischen Wichtigkeit und momentanem Förderlevel für die Lernkompetenz $(\Delta=1,83)$ und die Selbstbestimmungskompetenz $(\Delta=1,81)$ ergeben - zwei derjenigen Skills, die als mit am wichtigsten eingestuft worden waren. Im Unterschied hierzu wurde die Initiativ- und Leistungskompetenz sowohl als wichtig bewertet $(M=4,13, S D=0,89)$, als auch dass ihre Förderung laut Meinung der Expertinnen und Experten in akzeptablem Maße durch die Hochschulen gefördert $(M=3,07, S D=0,93)$.

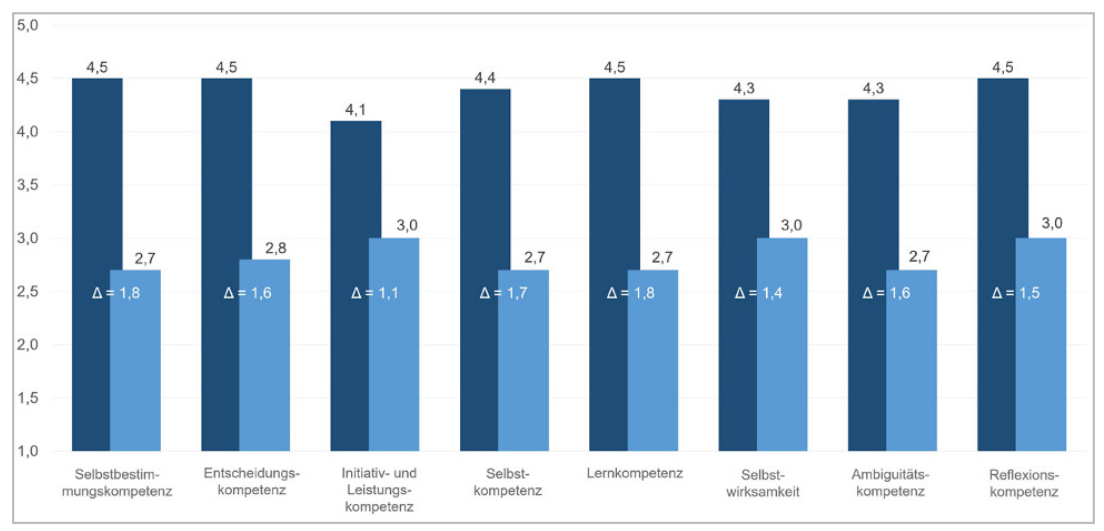

Abb. 15 Subjekt-entwicklungsbezogene Kompetenzen: Wichtigkeit versus Fähigkeit von Hochschulen Future Skills Entwicklung zu fördern $(\mathrm{N}=46)^{15}$

15 Die ebenfalls zu den subjekt-entwicklungsbezogenen Kompetenzen gehörige ethische Kompetenz wurde im Rahmen der Delphi-Studie nicht erfasst. 
Ein Blick auf die Diskrepanzen - dargestellt in Abbildung 16 - gibt Hinweise auf den Grad der Dringlichkeit, mit der Konzepte entwickelt werden müssen, um die Kompetenzentwicklung voranzutreiben. Die Kreise, welche die höchsten Deltas ausweisen zeigen gleichermaßen das Maß der höchsten Dringlichkeit auf (Abbildung 16 links), wohingegen die kleineren Deltas (Abbildung 16 rechts) auf weniger dringliche Aspekte schließen lassen.

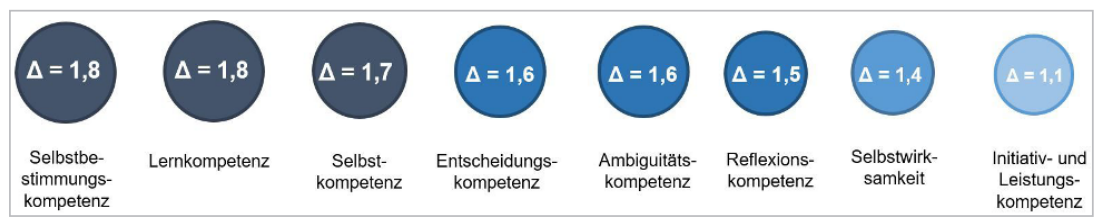

Abb. 16 Diskrepanzwerte für subjekt-entwicklungsbezogene Kompetenzen zwischen Skill Wichtigkeit und deren Förderung durch Hochschulen $(\mathrm{N}=46)^{16}$

\section{A 4.2 Reifegrad für individuell-objektbezogene Kompetenzen}

Individuell-objektbezogene Kompetenzen sind diejenigen Skills, die auf Fähigkeiten des Individuums basieren, in unbekannten zukünftigen Kontexten zu agieren, bei denen aber nicht das Individuum selbst den Bezugspunkt darstellt, sondern ein bestimmtes Objekt, auf welches sich die Handlung bezieht - beispielsweise eine bestimmte Aufgabe.

16 Die ebenfalls zu den subjekt-entwicklungsbezogenen Kompetenzen gehörige Ethische Kompetenz wurde im Rahmen der Delphi-Studie nicht erfasst. 


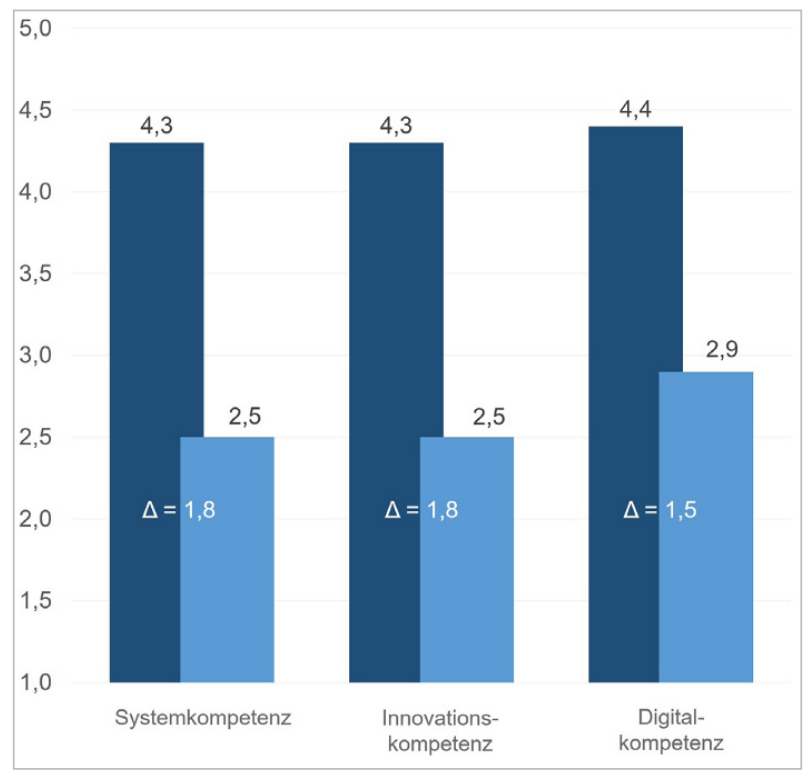

Abb. 17 Individuell-objektbezogene Kompetenzen: Wichtigkeit versus Fähigkeit von Hochschulen Future Skills Entwicklung zu fördern ${ }^{17}$

Das Expert(inn)ensample bewertete alle Skills dieser Kategorie als wichtig. Am wenigsten von Hochschulen gefördert werden - wie aus Abbildung 17 ersichtlich - nach Meinung der Expertinnen und Experten Agilität (Systemkompetenz) $(M=$ $2,53, S D=0,87)$ und Kreativität (Innovationskompetenz) $(M=2,52, S D=0,85)$. Dies schlägt sich für beide Kompetenzen in Form der höchsten Diskrepanz zwischen ihrer Wichtigkeit einerseits und der Förderung dieser Skills durch Hochschulen andererseits nieder (siehe Abbildung 18).

Die Delphi-Befragten bewerteten die Digitalkompetenz als in akzeptablem Maße gefördert $(M=2,93, S D=1,03)$. Mit Blick auf die Häufigkeitsverteilungen zeigt sich jedoch, dass immerhin 40 Prozent der Expertinnen und Experten den Reifegrad von Hochschulen diesen Skill bei ihren Absolventinnen und Absolventen erfolgreich auszubilden, als gering oder sogar sehr gering einschätzten. Dagegen halten 37,8 Prozent die Fähigkeit der Hochschulen in diesem Punkt für (sehr) gut.

17 Die ebenfalls zu den individuell-objektbezogenen Kompetenzen gehörige Design Thinking-Kompetenz wurde im Rahmen der Delphi-Studie nicht erfasst. 


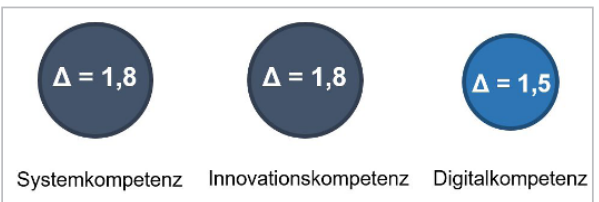

Abb. 18 Diskrepanzwerte für individuell-objektbezogene Kompetenzen zwischen Skill Wichtigkeit und deren Förderung durch Hochschulen $\left(\mathrm{N}_{\text {Importance }}=44\right.$, $\mathrm{N}_{\text {Support }}=45$ )

\section{A 4.3 Reifegrad für organisationsbezogene Kompetenzen}

Unter organisationsbezogenen Kompetenzen werden solche Skills gruppiert, die dazu benötigt werden, um in organisationalen und sozialen Umwelten erfolgreich agieren zu können.

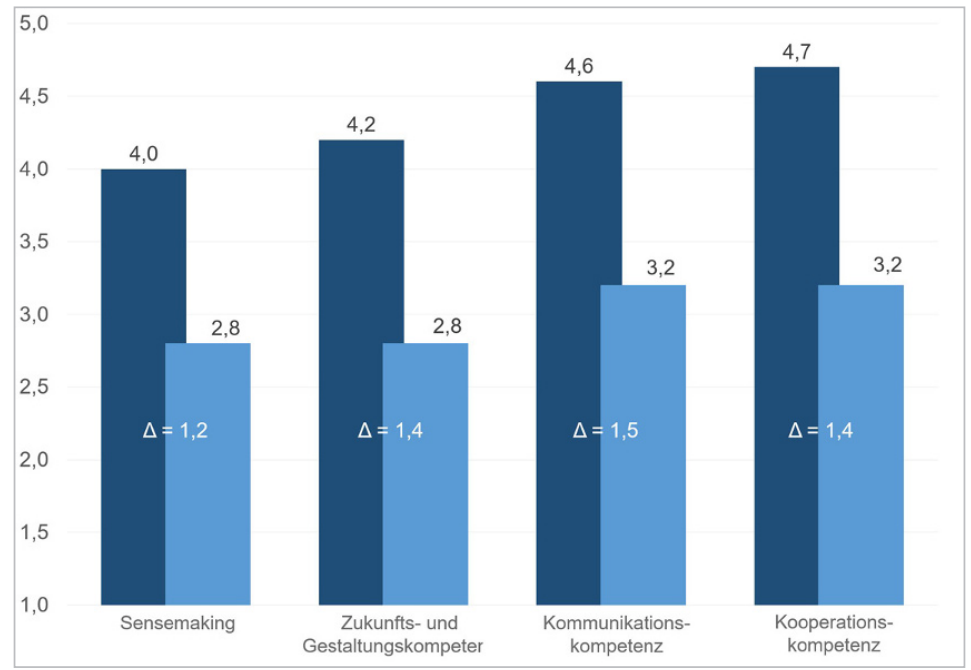

Abb. 19 Organisationsbezogene Kompetenzen: Wichtigkeit (dunkelblaue Balken) versus momentane Fähigkeit von Hochschulen Future Skills Entwicklung zu fördern (hellblaue Balken) $(\mathrm{N}=45)$ 
Auch in dieser Sektion bewertete das internationale Expert(inn)ensample alle Skills auf einem hohen Niveau als wichtig, wobei die Kooperationskompetenz mit einem Mittelwert von 4,59 $(S D=0,67)$ und die Kommunikationskompetenz mit einem Mittelwert von 4,67 (SD =0,67) sogar als sehr wichtig eingestuft wurden (siehe Abbildung 19). Zudem schätzten die Expertinnen und Experten alle Skills in dieser Kategorie als in akzeptablem Maße durch die Hochschulen gefördert ein, wobei die beiden als am wichtigsten erachteten Skills - die Kooperations- und die Kommunikationskompetenz - gleichzeitig auch als die am besten geförderten Future Skills überhaupt eingeschätzt wurden.

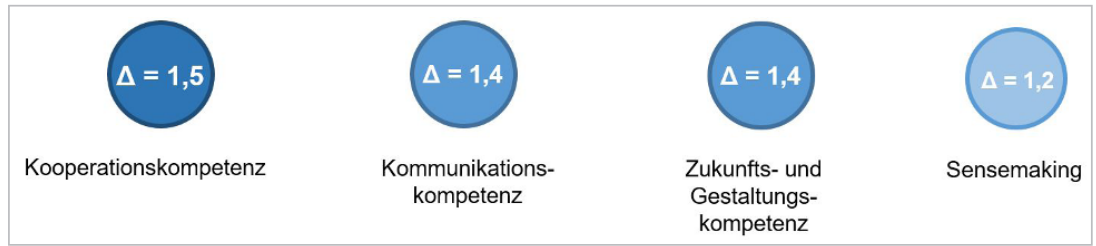

Abb. 20 Diskrepanzwerte für organisationsbezogene Kompetenzen zwischen Skill Wichtigkeit und deren momentaner Förderung durch Hochschulen $(\mathrm{N}=45)$

Die Expertinnen und Experten betonten, dass der Grad der Förderung von Future Skills zwischen den Hochschulen, den unterschiedlichen Hochschularten und auch in Abhängigkeit von Studiengängen und Lehrstilen Unterschiede aufweise. Auch Studierende seien in Abhängigkeit von Alter, Persönlichkeit und Einstellung unterschiedlich bereit zur Entwicklung von Future Skills. In einer vom Stifterverband beauftragten Studie zum Thema Future Skills werden strategische Potenziale für Hochschulen herausgestellt, mit denen die Defizite bei der Integration von Future Skills in die Hochschullehre verbessert werden sollen (Meyer-Guckel et al. 2019):

- Demnach stehen Hochschulen vor der Herausforderung, alle ihre Studierenden auf eine digitalisierte Arbeitswelt vorzubereiten. Das erfordert von Hochschulen neue Bildungsstrategien und eröffnet ihnen eine Reihe strategischer Potenziale. Derzeit mangelt es vor allem an Bildungsangeboten, die Zukunftskompetenzen vermitteln.

- Bei der Vermittlung von Zukunftskompetenzen werden Hochschulen für Unternehmen immer bedeutender: Schon heute arbeitet laut Stifterverband jedes vierte Unternehmen mit Hochschulen zusammen, um seine Kompetenzbedarfe zu decken. Tendenz steigend. 
- Hochschulen brauchen Innovationen und mehr Ressourcen in ganz unterschiedlichen Bereichen. Dazu zählen die Konzipierung neuer Studiengänge, die Weiterentwicklung bestehender Curricula, Vermittlung von Future Skills, die Schaffung neuer Lernumgebungen und agiler Innovationsräume sowie die Positionierung von Hochschulen als Weiterbildungsanbieter für lebenslange Lernprozesse. 